\title{
EFECTOS DE LIOFILIZACIÓN SOBRE COMPOSICIÓN QUÍMICA Y CAPACIDAD ANTIOXIDANTE EN PULPA DE CUATRO VARIEDADES DE Mangifera indica
}

*Felipe Surco-Laos ${ }^{1}$, Rocío Tipiana ${ }^{1}$, Ysabel Torres ${ }^{1}$, Manuel Valle, Juan Panay ${ }^{1}$.

\begin{abstract}
RESUMEN
El mango, Mangifera indica L., es una de las frutas tropicales que destaca por su particular sabor y aroma, tiene amplia aceptación. En el presente trabajo se evaluó el efecto de la liofilización sobre la pulpa de cuatro variedades de mango: Chato, Rosado, Carne y Chupar. Se efectuó un análisis químico bromatológico por métodos oficiales (AOAC, FAO) y la capacidad antioxidante por DPPH antes y post tratamiento. No se encontró diferencias significativas entre las variedades de mangos salvo en el contenido de vitamina $\mathrm{C}$ (rosado y carne $\sim 50 \%+$ ), y carotenoides (rosado $\sim 70 \%+$ ); el procesamiento afectó la acidez con un incremento de $250 \%$, una disminución de carotenoides totales (27- $42 \%$ ) y actividad antioxidante $(\sim 50 \%)$.
\end{abstract}

Palabras clave: Mango, liofilización, composición química, actividad antioxidante.

\section{LYOPHILIZATION EFFECTS ON CHEMICAL COMPOSITION AND ANTIOXIDANT CAPACITY IN PULP OF FOUR VARIETIES Mangifera indica}

\begin{abstract}
ABSTRAC
The mango, Mangifera indica L., is a tropical fruit that stands out for its particular flavor and aroma, is widely accepted. In this paper the effect of lyophilization on the pulp four mango varieties was evaluated: Chato, Pink, Meat and Suck. Bromatological chemical analysis by official methods (AOAC, FAO) and antioxidant capacity by DPPH before and after treatment was performed. No significant differences between varieties of mangos, except vitamin $\mathrm{C}$ (pink and meat $\sim 50 \%+$ ) and carotenoids (Pink $~ 70 \%+$ ) was found; the processing affected the acidity with an increase of $250 \%$, a decrease of total carotenoids (27-42\%) and antioxidant activity $(\sim 50 \%)$.
\end{abstract}

Key words: Mango, lyophilization, chemical composition, antioxidant activity.

${ }^{1}$ Facultad de Farmacia y Bioquímica UNICA. Ciudad Universitaria Ica.

*felipesurco@gmail.com 


\section{INTRODUCCIÓN}

El mango (Mangifera indica L.) se encuentra entre las frutas preferidas por la población en el país, entre las variedades iqueñas tenemos al mango rosado, mango chato, mango de carne, mango de chupar, variedades no injertadas o poliembriónicas de comercio interno en nuestro país ${ }^{1,2}$. En el 2013 la producción nacional de mango alcanzó un incremento de 196,5 por ciento en comparación con el mismo mes del año pasado, con un superávit en Ica de 29,2 por ciento ${ }^{1-3}$.

El consumo fresco del mango puede ser aprovechado de diversas formas, su disponibilidad se concentra en determinadas épocas durante el año por ello es considerado un fruto estacional y perecedero; este hecho debe constituir un incentivo para su conservación, allí la importancia de generar alternativas que prolonguen su vida útil.

El proceso de liofilización es el procedimiento de deshidratación más confiable en la conservación de las características sensoriales y nutricionales de un producto alimenticio, gracias al uso de bajas temperaturas y condiciones especiales de vacío, de ahí el objetivo del presente estudio de comprobar que el proceso de liofilización de la pulpa de mango no afectase su composición química y la actividad antioxidante de esta fruta ${ }^{3-5}$.

\section{PARTE EXPERIMENTAL}

\section{Material botánico}

Muestra de mango (Mangifera indica L.) de las diferentes variedades: Chato, Rosado, de Carne y de Chupar. Las cuales fueron adquiridas en los mercados de la ciudad de Ica entre los meses de febrero y marzo del 2014. Los análisis químicos fueron efectuados en los laboratorios de análisis instrumental y control de calidad, el proceso de liofilización en el laboratorio de tecnología farmacéutica de la Facultad de Farmacia y Bioquímica de la Universidad Nacional San Luis Gonzaga de Ica.

\section{Determinaciones analíticas}

Se realizó los análisis bromatológicos ante y post tratamiento de liofilización a la pulpa de las cuatro variedades de mango según la metodología de la $\mathrm{AOAC}^{6}$ y FAO ${ }^{7}$, los carbohidratos se determinó por diferencia. Para la determinación de carotenoides totales se realizó una extracción con alcohol isopropílico y lectura al espectrofotómetro a $460 \mathrm{~nm} 8$ y los resultados se expresan como $\beta$-carotenos, la actividad antioxidante se realizó por reducción del radical 1,1-difenil-2-picrilhidrazil (DPPH), con una absorbancia a 515nm.

El proceso de liofilización se llevó a cabo en un liofilizador marca Shuin, congelando la muestra a $-60{ }^{\circ} \mathrm{C}$ por 24 horas y un proceso de deshidratación a una presión de $900 \mathrm{mmHg}$ por 72 horas. 


\section{RESULTADOS Y DISCUSIÓN}

Tabla 1. Características de variedades de mango estudiadas

\begin{tabular}{|c|c|c|c|c|c|}
\hline $\begin{array}{l}\text { Variedades no } \\
\text { injertadas o } \\
\text { poliembriónicas }\end{array}$ & Tamaño & Forma & Color & Sabor & Cosecha \\
\hline Chato & Grande & $\begin{array}{l}\text { Notablemente } \\
\text { aplanada en un } \\
\text { extremo }\end{array}$ & $\begin{array}{l}\text { Amarillo } \\
\text { anaranjado } \\
\text { con chapa } \\
\text { rojiza }\end{array}$ & Agradable & Dic-Febr. \\
\hline Carne & Mediano & $\begin{array}{l}\text { Oval } \\
\text { redondo con una }\end{array}$ & Amarillo & Agradable & Febr-Mar. \\
\hline Rosado & Grande & $\begin{array}{l}\text { abertura al } \\
\text { medio }\end{array}$ & Amarillo & Agradable & Febr-Mar. \\
\hline Chupar & Pequeño & Oval & Amarillo & Agradable & Mar-Abr. \\
\hline
\end{tabular}

La producción se centra en los meses de verano, principalmente febrero-marzo, estas variedades son más pequeñas que las cultivadas en la costa norte de país, que presenta un color rojo-amarillo y cuya producción es entre los meses noviembre a diciembre, principalmente $(\text { AGROBANCO - INIA) })^{3,5}$.

Tabla 2. Rendimiento porcentual de pulpa según variedades

\begin{tabular}{ccccc}
\hline \multirow{2}{*}{ Porción } & \multicolumn{4}{c}{ Variedad de mango } \\
& Chato & Carne & Rosado & Chupar \\
\hline $\begin{array}{c}\text { Peso fruta } \\
\text { Peso de } \\
\text { cáscara }\end{array}$ & $246,84 \pm 19,2$ & $167,44 \pm 37,9$ & $224,96 \pm 45,8$ & $127,92 \pm 26,0$ \\
Peso de pepa & $51,34 \pm 8,25$ & $30,35 \pm 5,46$ & $42,93 \pm 11,2$ & $33,60 \pm 5,84$ \\
& & & & \\
Peso de pulpa & $162,55 \pm 15,6$ & $112,32 \pm 32,2$ & $149,99 \pm 31,4$ & $73,85 \pm 18,03$ \\
$\begin{array}{c}\text { Rendimiento } \\
\text { promedio \% }\end{array}$ & $65,32 \pm 3,92^{\mathrm{a}}$ & $66,19 \pm 5,42^{\mathrm{a}}$ & $67,73 \pm 2,73^{\mathrm{a}}$ & $57,47 \pm 4,59^{\mathrm{b}}$ \\
\hline
\end{tabular}

Letras superíndices iguales significa que no hay diferencia estadística significativa

Se representa el peso promedio de 10 unidades, resultando el más pequeño la variedad de chupar; sin embargo, todas estas variedades serían pequeñas comparadas con las variedad injertadas (peso oscila entre $600 \mathrm{a} 800 \mathrm{~g}$ ). Las variedades chato, carne y rosado no presentaron diferencia significativa en su rendimiento de pulpa, estando muy cerca al rendimiento de variedades injertadas $(70 \text { a } 75 \%)^{9}$. El chato y rosado se utilizan para elaboración de pulpa y jugos concentrados ${ }^{5}$. 
Tabla 3. Rendimiento de la liofilización de pulpa de mango

\begin{tabular}{lcccc}
\hline \multicolumn{1}{c}{ Porción } & \multicolumn{4}{c}{ Variedad de mango } \\
& Chato & Carne & Rosado & Chupar \\
\hline & & & & \\
& & & & \\
\hline $\begin{array}{l}\text { Peso promedio de } \\
\text { fruta fresca (g) }\end{array}$ & $19,11 \pm 2,41$ & $21,90 \pm 2,96$ & $20,09 \pm 0,62$ & $24,11 \pm 1,92$ \\
$\begin{array}{l}\text { Peso promedio de } \\
\text { fruta liofilizada (g) }\end{array}$ & $6,16 \pm 0,26$ & $7,46 \pm 0,64$ & $8,01 \pm 0,11$ & $7,48 \pm 0,82$ \\
$\begin{array}{l}\text { Rendimiento } \\
\text { promedio \% }\end{array}$ & $32,59 \pm 3,47^{\mathrm{a}}$ & $34,17 \pm 1,68^{\mathrm{a}}$ & $33,24 \pm 0,40^{\mathrm{a}}$ & $31,05 \pm 2,65^{\mathrm{a}}$ \\
\hline
\end{tabular}

Valor promedio de tres repeticiones. Letras superíndices iguales significa que no hay diferencia estadística significativa

Como se puede apreciar el rendimiento del proceso de liofilización no presentó diferencias con respecto a la variedad de mango, siendo la variedad de chupar ligeramente menor en su rendimiento. El rendimiento depende del tipo de fruta y el proceso aplicado ${ }^{10-12}$.

Tabla 4. Análisis químico proximal de pulpa fresca, según variedades de mango

\begin{tabular}{ccccc}
\hline $\begin{array}{c}\text { Análisis } \\
\text { proximal }\end{array}$ & \multicolumn{4}{c}{ Variedades de mango } \\
Chato & Carne & Rosado & Chupar \\
\hline $\begin{array}{c}\text { Humedad g/100 g } \\
\text { Cenizas g/100 g }\end{array}$ & 78,90 & 76,58 & 74,26 & 78,71 \\
Grasa g/100 g & 0,77 & 0,69 & 0,72 & 0,58 \\
Proteína g/100 g & $1,53^{\mathrm{a}}$ & 1,06 & 1,04 & $1,55^{\mathrm{a}}$ \\
$\mathbf{p H}$ & 4,00 & 4,04 & 4,29 & 4,77 \\
Acidez g/100 g & 0,5 & 0,4 & 0,35 & 0,2 \\
Sólidos solubles g/100 g & 17,4 & 19,8 & 22,2 & 18,7 \\
Carbohidratos g/100 g & 18,69 & 21,58 & 23,88 & 19,07 \\
\hline
\end{tabular}

Letras superíndices iguales significa que no hay diferencia estadística significativa 
En el análisis químico proximal de la pulpa de las cuatros variedades no se aprecia diferencia significativa más que en solo en dos parámetros: proteína que en las variedades chato y de chupar supera en promedio $50 \%$ a las otras dos y la acidez presentó diferencias en las cuatro variedades, las cuales estuvieron entre $0,2-0,5$; sin embargo, todos estos valores se encuentra dentro del rango de variación que reporta Ramírez et al., $2010^{13}$ para trece cultivares de mango en Venezuela. Se puede observar que existe una correlación entre menor acidez y mayor valor de $\mathrm{pH}$ en los cuatro tipos de mangos. Asimismo, se debe tener en cuenta que la variedad rosado presentó un mayor contenido de sólidos solubles (Brix 22.2) que lo reportado en otros estudios ${ }^{9}$, que implicaría un mayor grado de madurez; los valores de carbohidratos se han obtenido por cálculos, por lo que pequeñas diferencias en los otros parámetros determinados (humedad, proteína, cenizas y grasas) se expresen en éste como una diferencia significativa.

Tabla 5. Análisis químico proximal de pulpa liofilizada según variedades de mango

\begin{tabular}{ccccc}
\hline $\begin{array}{c}\text { Análisis } \\
\text { proximal }\end{array}$ & Chato & Carne & Rosado & Chupar \\
\hline Humedad g/100 g & 2,32 & 3,34 & 2,81 & 2,72 \\
Cenizas g/100 g & 3,41 & 2,54 & 2,35 & 2,32 \\
Grasa g/100 g & $0,75^{\mathrm{b}}$ & $0,46^{\mathrm{a}}$ & $0,47^{\mathrm{a}}$ & $0,4^{\mathrm{a}}$ \\
Proteína g/100 g & 7,62 & 6,27 & 4,93 & 6,87 \\
$\mathbf{p H}$ & 3,58 & 3,52 & 3,74 & 3,89 \\
Acidez g/100 g & $2,5^{\mathrm{a}}$ & $2,4^{\mathrm{a}}$ & $2,16^{\mathrm{b}}$ & $1,69^{\mathrm{c}}$ \\
\begin{tabular}{c} 
Carbohidratos $\mathbf{~ g / 1 0 0 ~ g}$ \\
\hline
\end{tabular} & 85,90 & 87,39 & 89,44 & 87,69 \\
\hline
\end{tabular}

Letras superíndices iguales significa que no hay diferencia estadística significativa.

La muestra de pulpas liofilizadas al análisis químico bromatológico no presentaron diferencias significativas entre ellas salvo en la determinación de grasa, la cual se puede atribuir a un error analítico. Al convertir los datos de muestra fresca en base seca y comparar con las liofilizadas, únicamente se puede apreciar diferencia significativa en la determinación de acidez. Se debe tener en cuenta que las frutas contienen ácidos orgánicos de forma libre o combinada $^{11,14}$ que están disueltos en las vacuolas de las células que al eliminar el agua se concentrarían. 


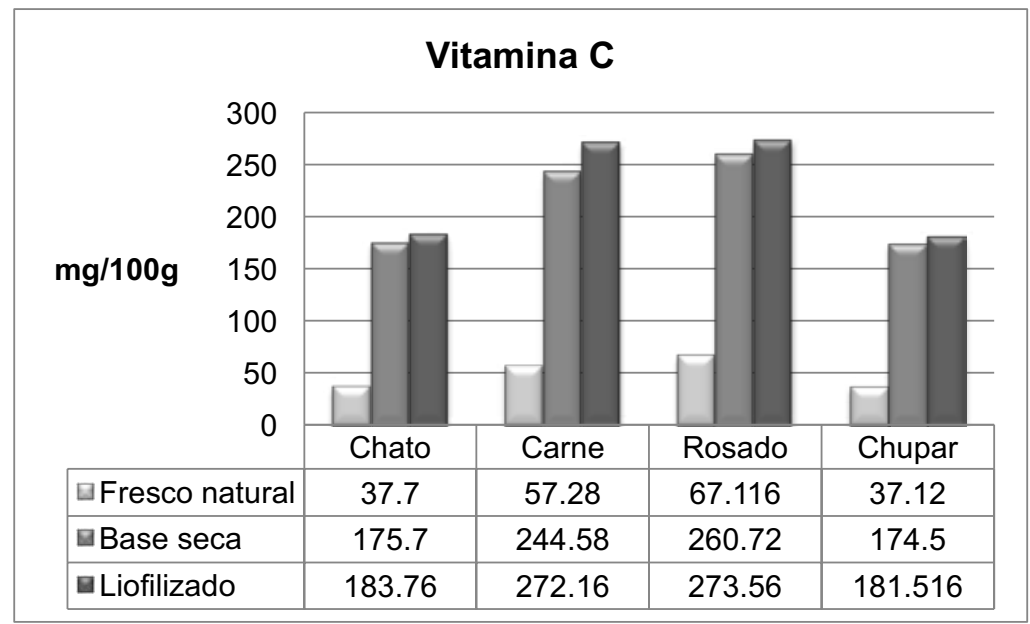

Figura 1. Contenido de vitamina $\mathrm{C}$ en pulpa de mango según variedad

La variedad carne y rosado muestran un mayor contenido de vitamina $\mathrm{C}$ al estado fresco, con valores superiores a lo declarado en la tabla peruana de composición de alimentos para el mango, pudiéndose clasificar a esta fruta como una excelente fuente de vitamina $\mathrm{C}$. El proceso se liofilización no afecta significativamente el contenido de vitamina $\mathrm{C}$, estos datos son concordantes con los de otros autores a otras variedades de frutas (Huaraca et al. $2010^{11} \mathrm{y}$ Amores et al. 2011 ${ }^{12}$ ) los cuales manifiestan que el proceso de liofilización permite preservar este componente.

Tabla 6. Relación del contenido de carotenoides totales (mg ß-caroteno/100 g)

\begin{tabular}{|c|c|c|c|}
\hline Variedad & Pulpa fresca (b.s) & Pulpa liofilizada & $\%$ pérdida \\
\hline Chato & 238,30 & 140,9 & 40,9 \\
\hline Carne & 169,32 & 122,78 & 27,5 \\
\hline Rosado & 1010,20 & 645,42 & 36,1 \\
\hline Chupar & 269,05 & 172,64 & 35,8 \\
\hline
\end{tabular}

b.s. $=$ base seca.

El contenido de carotenoides totales se vio afectado considerablemente con el proceso de liofilización (congelación a $-60{ }^{\circ} \mathrm{C}$ y deshidratado por 72 horas) en todas variedades de mango, siendo la más afectada la variedad carne con una pérdida de 40,9\%. Esto se vio reflejado en la pérdida de color del liofilizado ${ }^{16}$. 
Tabla 7. Determinación de la capacidad de inhibición del radical DPPH

\begin{tabular}{|c|c|c|c|c|}
\hline \multirow[b]{2}{*}{$\begin{array}{l}\text { Variedades } \\
\text { de mango }\end{array}$} & \multicolumn{2}{|c|}{ Pulpa fresca (b.s.) } & \multicolumn{2}{|c|}{ Pulpa liofilizada } \\
\hline & $\begin{array}{c}\text { Concentración } \\
\text { mg/ ml }\end{array}$ & $\begin{array}{c}\text { \% Inhibición } \\
\text { DPPH }\end{array}$ & $\begin{array}{c}\text { Concentración } \\
\mathrm{mg} / \mathrm{ml}\end{array}$ & $\begin{array}{c}\text { \% Inhibición } \\
\text { DPPH }\end{array}$ \\
\hline Chato & 12,39 & 32,26 & 12,05 & 16,12 \\
\hline Carne & 10,73 & 61,69 & 10,7 & 30,55 \\
\hline Rosado & 13,42 & 32,05 & 12,2 & 28,7 \\
\hline Chupar & 10,44 & 40,08 & 10,2 & 14,94 \\
\hline
\end{tabular}

La pérdida de la actividad antioxidante en las distintas variedades de pulpa de mango, podemos atribuirla principalmente a la pérdida de carotenoides totales que son sustancias con reconocida actividad antioxidante14,15.

\section{CONCLUSIONES}

- La composición químico proximal de las pulpa fresca de las cuatros variedades de mango no presenta diferencias significativas; sin embargo, en cuanto a los compuestos bioactivos, la variedad rosado presenta mayor contenido de vitamina $\mathrm{C}$ y carotenoides.

- En el análisis químico proximal de la pulpa liofilizada se puede ver diferencia significativa en el mayor contenido de grasa en la variedad chato y un menor contenido de proteína en la variedad rosado, con respecto a sus compuestos bioactivos, menor contenido de vitamina $\mathrm{C}$ y carotenoides en la variedad chato, pero mayor actividad antioxidante en la variedad carne y rosado.

- Con respecto al efecto del proceso de liofilización afectó significativamente parámetros como: el contenido de acidez que se incrementó y una reducción apreciable en el contenido de carotenoides y actividad antioxidantes en todas las variedades de mango.

\section{BIBLIOGRAFÍA}

1. Ica, Tierra de Paracas, Pallares Pisco y Pecanas [Internet]; 2009. [Acceso 12 Ene 2014]. Disponible en: http://www.senoriodesulco.com/docs/articulos/art_9_ica_tierra_de_ paracas_pallares_piscos_y_pecanas.pdf

2. Ministerio de Salud-Instituto Nacional de Salud. Tabla Peruana de Composición de los Alimentos. Lima-Perú: INS; 2009.

3. Ministerio de Agricultura. Oficina General de Planificación Agraria. Perfil de Mercado del Mango. Unidad de Comercio internacional. 2006. [Acceso 15 de junio 
2014]. Disponible en: http://www.prompex.gob.pe/Miercoles/Portal/MME/descargar. aspx archivo $=$ CCF5075F-376A-4070-ACA1-FF27BB9171A4.PDF

4. Instituto Nacional de Investigación Agraria. Variedades de Mango. [Tríptico]. Lima: INIA; 2008.

5. MINCETUR. Perfil del Mercado y Competitividad Exportadora de Mango [Internet]. [Acceso 24 de noviembre 2013]. Disponible en: http://bibliotecavirtual.corpmontana. com/handle/123456789/168?show=full

6. AOAC. Official Methods of Analysis. 18th ed. Gaithersburg: Association the Official Agricultural Chemists; 2007.

7. FAO. Manuals of food quality control. Food and nutrition paper 14/7. Rome: FAO; 1986.

8. Britton, G. Carotenoids and Colors in Fruits and vegetables. Phytochemistry of fruit and vegetables, eds. Clarendon Press, Oxford, pp.11 -27 (1997).

9. Marulanda J. Determinación del perfil de calentamiento y evaluación sensorial en la elaboración de pulpa liofilizada de mango variedad Tommy Atkins. [Tesis]. ManizalesColombia: Universidad Nacional de Colombia. Facultad de Ciencia y Tecnología de los Alimentos; 2002. [Acceso 24 de noviembre 2013]. Disponible en: http://www.bdigital. unal.edu.co/1009/1/jorgemariomarulandaloaiza.2002.pdf

10. Natividad L, Cáceres J. Algunos aspectos técnicos sobre la liofilización de pulpa de cocona (Solanum sessiliflorum Dunal). RVCTA. 2013; 4 (2): 207-218.

11. Huaraca A. Evaluación nutritiva y nutracéutica de la frutilla (Fragaria vesca) deshidratada por el método de liofilización y comparación con la obtenida por deshidratación en microondas. Riobamba - Ecuador: Escuela Superior Politécnica de Chimborazo. Facultad de Ciencias - Escuela de Bioquímica y Farmacia; 2011.

12. Amores D. Evaluación Nutritiva y Nutraceútica de la Mora de Castilla (Rubus glaucus) Deshidratada por el Método de Liofilización y Comparación con la obtenida por Deshidratación en Microondas y Secador en bandejas. [Tesis]. Riobamba - Ecuador: Escuela Superior Politécnica de Chimborazo. Facultad de Ciencias - Escuela de Bioquímica y Farmacia; 2011. [Acceso 20 de mayo 2014]. Disponible en:http://dspace. espoch.edu.ec/bitstream/123456789/1989/1/56T00297.pdf

13. Ramírez R, Quijada O, Castellano G, Burgos M, Camacho R, Marin C. Características Físicas y Químicas de Frutos de Trece Cultivares de Mango (Mangifera indica L) en el Municipio Mara en la Planice de Maracaibo. Rev Iber Tecnología Postcosaecha. 2010; 10(2): 65-72.

14. Perkins P, Manthey J. Explorando Los Fitoquímicos del Mango Importado. [Internet]. Servicio de Investigación Agrícola de la USDA; 2007. [Acceso 20 de Junio 2014]. Disponible en:

http://www.mango.org/Mangos/media/Media/Documents/Research\%20And\%20Resources/ Research/Industry/Nutrition/Fitoquimicos-del-mango-importado-Reporte-1.pdf

15. Salamanca G, Forero F, García J, Díaz C. Avances en la Caracterización, Conservación y Procesamiento del Mango (Mangifera indica L.). Revista Tumbaga. 2007; 2: 57-64.

16. Ingeniería en Industrias Alimentarias / Instituto Tecnológico Superior de Calkiní. Deshidratación: secado y liofilización. [Articulo virtual]; 2011 [Acceso 15 de febrero 2014]. Disponible en: https://www.revistavirtualpro.com/biblioteca/deshidratacionsecado-y-liofilizacion. 